spleen with 4 weeks of induction therapy. The potent effect of SINE compound monotherapy on GC and auto-reactive ASC was further highlighted by the pronounced elimination of GCs histologically and reduction in auto-reactive ASC achieved after 4 weeks of maintenance therapy administered once weekly. In a concurrent study, when combined with bortezomib, 1 week verdinexor plus PI treatment resulted in a synergistic effect, significantly reducing in the number of autoreactive ASC, particularly in the BM.

Conclusions Verdinexor has demonstrated efficacy by reducing generation and survival of auto-reactive immune cells. Additional experiments are underway to examine if inhibition of the canonical NFB pathway underlies verdinexors inhibitory effect. Together, our findings suggest the potential of SINE compounds to have a significant impact on SLE disease progression alone or in combination with currently utilized PIs.

Funding Source(s): NIH R44 AI124949-03

\section{CLINICAL CHARACTERISTICS OF SYSTEMIC LUPUS ERYTHEMATOSUS WITH MALIGNANT TUMORS}

Honglian Yu. Shuangyi Campus of Ningxia Medical University

\subsection{6/lupus-2019-Ism.229}

Background Rheumatic disease is caused by immune abnormality, which results in the immune response of the body to autoantigens and causes tissue damage.The immune system eliminates or inhibits the growth of tumor cells through various ways. When the immune function abnormality leads to the escape of immune surveillance of tumor cells, it can lead to tumorigenesis. The risk of malignant tumors is higher than ordinary people in systemic lupus erythematosus (SLE) patients, which were mainly hematological tumors,especially non-Hodgkin lymphoma.The risk of Hodgkin lymphoma, leukemia and multiple myeloma was also increased, which was associated with BAFF changes,APRIL signal transduction, increased IL-6 activity and EB virus infection.Increased risk of malignant tumors in SLE patients,including lung cancer,hepatobiliary cancer,thyroid cancer, vulvar/vaginal malignancies and cervical dysplasia,was also reported,which was associated with SLE disease activity in specific organs, inflammatory stimulation,oxidative damage, changes in viral clearance and/or specific drug use.

Methods Twenty SLE patients with malignant tumors admitted to the General Hospital of Ningxia Medical University from October 2008 to October 2018 were selected.Their sex, age,time of diagnosis of rheumatism and tumors, types of tumors, clinical stages and prognosis were summarized and analyzed.

Results Malignant tumors occurred in 20 (0.50\%) of 3970 SLE patients, the average age was $54.43 \pm 7.95$. There were 4 cases of lung cancer, 3 cases of non-Hodgkin lymphoma and liver cancer, 2 cases of thyroid cancer,uterine cancer and skin cancer,Multiple myeloma, gastric cancer, pancreatic cancer and nasopharyngeal carcinoma were found in 1 case respectively. The median course of malignant tumors in SLE patients was 3.42 years.79.41\% of the patients were diagnosed before tumors, $16.35 \%$ of the cases occurred at the same time, And only $4.24 \%$ of tumors were diagnosed before SLE.Phase IV was the most common clinical stage in the diagnosis of tumors, accounting for 59.74\%.In SLE patients with tumors, the survival time ranged from 1 month to 39 months, with a median survival time of 9.2 months.

Conclusions Most malignant tumors are found after SLE diagnosis, with a median course of 3.42 years, and occur more frequently between the ages of 50 and 60.SLE may be associated with various types of malignant tumors, Lung cancer, non-Hodgkin's lymphoma and Liver cancer were the most common tumors, and the clinical staging of tumors is late and the prognosis is poor,with the median survival time is 9.2 months.

Funding Source(s): None

\section{SYSTEMIC LUPUS ERYTHEMATOSUS AND RAPIDLY PROGRESSIVE CARDIOGENIC SHOCK}

Anam Umar*, Eric Chang, Muhammad Bilal, Cinnamon Bradley. Morehouse School of Medicine

\subsection{6/lupus-2019-Ism.230}

Background Symptomatic lupus myocarditis is a rare but lifethreatening complication of systemic lupus erythematosus (SLE). Clinical manifestation is variable and includes dyspnea, chest pain, peripheral edema, fever, nausea, vomiting, or palpitations. We describe a case of a young female who developed rapid progressive cardiogenic shock secondary to lupus myocarditis.

Methods Not applicable as it is a clinical vignette.

The abstract for clinical vignette is attached as a separate document

Results Not applicable as it is a clinical vignette

The abstract for clinical vignette is attached as a separate document

Conclusions The diagnosis of lupus myocarditis is usually achieved clinically and with the use of TTE, cardiac MRI or biopsy. However, none of the current diagnostic modalities have established sensitivity or specificity. Our patient only developed significant TTE findings during the late stage, which led to an unfortunate delay in diagnosis. We suggest that physicians should always have a broad differential diagnosis and high clinical suspicion to avoid late diagnosis and mismanagement of patients, especially when dealing with cardiac manifestations of SLE.

Funding Source(s): None

\section{TRNA DERIVED FRAGMENTS(TRFS) REGULATE OXIDATIVE PHOSPHORYLATION TO PARTICIPATE IN SLE PATHOGENESIS}

'Guannan Geng, ${ }^{2}$ Huijing Wang ${ }^{*},{ }^{3}$ Shuang Ye. 'Renji Hospital, South campus, School of Medicine, Shanghai Jiao Tong University; ${ }^{2}$ Department of Rheumatology, South Campus, Renji Hospital, School of Medicine, Shanghai Jiaotong University, Shanghai, China; ${ }^{3}$ Department of Rheumatology, South Campus, Ren Ji Hospital, School of Medicine, Shanghai Jiao Tong University

\subsection{6/lupus-2019-Ism.231}

Background tRNA derived fragments(tRFs) are 18 to 40 nucleotide(nt) small RNAs cleaved from mature tRNAs or precursor tRNA transcripts. There is growing evidence that tRFs play important roles in cellular homeostasis. This study focused on abnormal expression of tRFs in CD4 + T lymphocytes from SLE patients, their function on 
regulation of oxidative metabolism and potential roles in development of systemic lupus erythematosus (SLE).

Methods CD4 + T lymphocytes isolated from 97 SLE patients, 30 RA patients, 10 AS patients and 20 healthy donors were used in our in vitro experiments. Total RNA of CD4 + T lymphocytes isolated from 4 SLE patients and 3 healthy donors were used to perform small RNA sequencing. Total RNA of CD4 + T lymphocytes transfected with synthetic tRF-3009 or negative control (a random sequence, single strand) were used to perform next generation sequencing. CD4 + T lymphocytes isolated from healthy donors were transfected with tRF-3009/negative control or tRF-3009 siRNA/si-NC in vitro, with/without IFN-alpha treatment, to analyze OCRROS and ATP concentration. Real-time qRT-PCR was performed to analyze expression of tRF-3009 and related gene expressions.

Results We have identified a series of tRFs expressed abnormally in SLE CD4 + T lymphocytes. Interestingly, almost all up regulated tRFs were from 3 end of mature tRNA while down regulated ones were from 3 end of tRNA precursors. We have found that expression of tRF-3009 was corelated with lupus nephritis (LN) and urine protein (PRO). The expression of tRF-3009 could be induced by IFN-alpha treatment in vitro. Knockdown of tRF-3009 by siRNA could rescue the metabolism change of $\mathrm{CD} 4+\mathrm{T}$ lymphocytes induced by IFN-alpha. Transfection of tRF-3009 alone could up regulate oxidative phosphorylation of CD4 + T lymphocytes in vitro.

Conclusions This study identified a series of abnormally expressed tRFs existed in CD4 + T lymphocytes isolated from SLE patients. One of these small RNAs, tRF-3009 also enriched in kidney and corelated with occurrence of lupus nephritis. tRF-3009 anticipated in metabolism regulation, may play important roles in SLE pathogenesis.

Funding Source(s): The National Key Research and Development Program of China(2016YFC0903900 and 2017YFC0909000)

\section{PREDICTORS OF HYDROXYCHLOROQUINE TREATMENT ADHERENCE AMONG PEOPLE WITH LUPUS}

Karin Tse ${ }^{*}$, Rossi Paola Daly. Lupus Foundation of America

\subsection{6/lupus-2019-Ism.232}

Background Treatment adherence is associated with better disease control, improved morbidity and mortality and increased quality of life. ${ }^{1}$ Studies examining treatment adherence predictors have varied in examined characteristics and results. This study analyzes demographic and socioeconomic factors, perceptions, and experiences influencing hydroxychloroquine (HCQ) treatment adherence among people with lupus. HCQ has long been used to treat lupus, and has been shown to reduce damage accrual and has protective effects on diseases such as diabetes and hyperlipidemia as well as overall survival in people with lupus. ${ }^{2}$

Methods Cross-sectional study data was obtained through the 2015-2016 Lupus Needs Survey, an online national needs assessment among 3022 adults self-reporting a lupus diagnosis. Univariate analyses were conducted using chi-square and simple logistic regressions among 21 variables, which were subsequently dichotomized. Variables with rates of missing values $>15 \%$ of total sample were removed in further analysis.
Abstract 232 Table 1 Results of Binary Logistic Model of Predictors of HCQ Treatment Adherence

\begin{tabular}{|c|c|c|c|}
\hline & Odds Ratio & $\begin{array}{c}95 \% \\
\text { Confidence } \\
\text { Interval }\end{array}$ & $p$-value \\
\hline Black & 0.35 & $0.18-0.67$ & $0.002^{*}$ \\
\hline $\begin{array}{l}\text { Other (mixed, American Indian/Alaskan Native, } \\
\text { Pacific Islander, or Middle Eastern) }\end{array}$ & 0.35 & $0.14-0.85$ & 0.021 * \\
\hline Moderate severity of lupus at diagnosis & 0.57 & $0.31-1.03$ & 0.061 \\
\hline HCQ improves/helps treat my symptoms & 2.49 & $1.25-4.92$ & $0.009^{*}$ \\
\hline Positive impression of HCQ & 2.14 & $1.08-4.24$ & $0.029^{*}$ \\
\hline Rheumatologist as primary doctor treating lupus & 2.0 & $1.01-3.95$ & $0.046^{*}$ \\
\hline
\end{tabular}

Next, 13 variables with a $\mathrm{p}$-value $<0.20$ were entered into a binary logistic model using backwards stepwise selection.

Results 2028 individuals reported currently taking HCQ with $1581(78.0 \%)$ individuals reporting taking the medication exactly as prescribed all of the time, 385 (19.0\%) reporting some of the time, and $62(3.1 \%)$ reporting sometimes or never taking HCQ as prescribed. People with lupus were twice as likely to adhere to HCQ if they reported having a rheumatologist as the primary doctor treating their lupus $(p=0.046, O R=2.00)$ and more than twice as likely if they reported having a positive impression of HCQ $(p=0.029$, $\mathrm{OR}=2.14$ ) or experienced improvement in treating their symptoms using HCQ $(p=0.009, \mathrm{OR}=2.49)$. Notably, African Americans and those indicating race as Other (mixed, American Indian/Alaskan Native, Pacific Islander or Middle Eastern) were $65 \%$ less likely to adhere to HCQ treatment $(p=0.002$, $\mathrm{OR}=0.35 ; \mathrm{p}=0.021, \mathrm{OR}=0.35$, respectively).

Conclusions The study confirms the importance of experiential factors such as medication impression and patient-reported symptom improvement in HCQ treatment adherence. Additionally, results highlight the potential significance of having a specialist as the primary lupus doctor. This may be due to rheumatologists higher awareness of the value of HCQ for lupus patients compared to other providers. Further exploration is needed on cultural factors negatively influencing treatment adherence among certain racial groups, particularly African Americans and Other racial groups (mixed, American Indian/Alaskan Native, Pacific Islander or Middle Eastern). Treatment adherence is particularly important in lupus given the complex nature of the disease and fluctuation of disease activity, which may be exacerbated by poor adherence.

Funding Source(s): UCB Pharma funded study data collection.

\section{AUTOANTIBODY-POSITIVE HEALTHY INDIVIDUALS CONSTRAIN T CELL PATHWAYS TO REGULATE AUTOIMMUNE DISEASE}

'Samantha Slight-Webb, 'Aleksandra Bylinska, ${ }^{1}$ Miles Smith, ${ }^{1}$ Rufei Lu, ${ }^{1}$ Hua Chen, ${ }^{1}$ Krista Bean, ${ }^{1}$ Melissa Munroe, ${ }^{2}$ Holden Maecker, ${ }^{2}$ Paul J Utz, ${ }^{1}$ Joan T Merrill, ${ }^{3}$ Eliza Chakravarty, ${ }^{1}$ Cristina Arriens, ${ }^{1}$ Joel Guthridge, ${ }^{1}$ Judith A James*. ' Oklahoma Medical Research Foundation; ${ }^{2}$ Stanford University; ${ }^{3}$ Oklahoma Medical Research Foundation; University of Oklahoma

\subsection{6/lupus-2019-Ism.233}

Background Anti-nuclear antibody (ANA) positivity is a principal feature of individuals with an autoimmune disease, yet up to one in five healthy individuals are ANA-positive (ANA+) 